\title{
The Potential Use of Monoclonal Antibodies as Therapeutic Modalities in Neonatal Infection
}

\author{
Harry R. Hill, ${ }^{*, 1}$ Luis Antonio Gonzales, ${ }^{1}$ \\ Douglas K. Kelsey ${ }_{9}^{2}$ and Howard V. Raff ${ }^{3}$ \\ 'Departments of Pathology and Pediatrics, University of Utah, \\ Salt Lake City, UT; ${ }^{2}$ The Mead Johnson Research Center, \\ Evansville, IN; and ${ }^{3}$ Bristol-Myers Squibb, Seattle, WA
}

Group B streptococcal (GBS) and Escherichia coli infections are serious problems in human neonates. It is estimated that 11,000 cases of neonatal GBS disease occur per year in the US, resulting in 2600 deaths (1). In most series, GBS and $E$. coli account for approximately equal numbers of cases of neonatal sepsis and meningitis. Thus, over 20,000 cases of neonatal disease and 4000-5000 deaths occur per year owing to these two major bacterial pathogens. Approximately one-half of the survivors suffer significant sequelae. Thus, neonatal sepsis and meningitis are major health problems in this as well as in other countries throughout the world. Attempts have been made to improve methods for detecting and treating or preventing these infections, but to date these have not gained wide enough use to affect the problem significantly. The lack of opsonic antibody in human neonates is one major risk factor for the development of bacterial infection $(2,3)$. In 1978, we (4) showed that the administration of fresh whole-blood transfusions, containing opsonic antibody, could be effective in decreasing mortality from GBS infection in human neonates. It was impossible to predict, however, whether blood donors would possess antibody to the bacterial strains causing infec-

*Author to whom all correspondence and reprint requests should be addressed.

IVIG Therapy Today - Ed.: M. Ballow - 1992 The Humana Press Inc. - Totowa, NJ 
tion. Furthermore, blood transfusion has significant risks, including transmission of infectious agents, such as hepatitis, cytomegalovirus, and, more recently, the human immunodeficiency virus. Because of these problems, our group as well as others began to explore the possibility of using intravenous immunoglobulin (IVIG) in the therapy of experimental neonatal infection $(5,6)$. These preparations had reasonable activity against less virulent GBS and $E$. coli strains, but almost no activity against more virulent strains, which produce or contain an excess of sialic acid in type-specific GBS or $E$. coli $\mathrm{K} 1$ antigens $(7,8)$. Furthermore, significant lot-to-lot variation in antibody concentrations among the available IVIG preparations was detected (9). For these reasons, our group turned to the developing technology of making hybridoma antibodies as a way of producing more efficacious preparations for use in neonatal bacterial infection.

\section{Methods}

Murine monoclonal antibodies (MuMAbs) were made using modifications of the methods of Galfre and colleagues (10). Mice were immunized with a virulent strain of type III GBS. Spleen cells from these animals were harvested and fused with SP 2/0 nonsecreting murine myeloma cells in the presence of polyethylene glycol. Following culture in hypoxanthine, aminopterine, thymidine medium (HAT), the culture supernatants were screened for antibody production against whole GBS. Positive hybridomas were cloned by limiting dilution and examined for opsonic and protective activity.

Hybridomas were injected intraperitoneally into Balb/c mice and high-titered ascitic fluid was obtained, from which IgM monoclonal antibody was purified (11). IgG2a and IgA murine monoclonal GBS type-III-specific antibodies were produced by colleagues at the University of Alabama, Birmingham (12).

IVIG from different manufacturers was employed in various aspects of these studies, including Gamimune N (Cutter Biologicals, Berkeley, CA; pH 4.25; 5\% immunoglobulin in 10\% maltose) and Sandoglobin ${ }^{\mathrm{TH}}$ (Sandoz Pharmaceuticals, East Hanover, NJ; pH 6.6; $6 \%$ immunoglobulin in $10 \%$ sucrose).

Human monoclonal antibodies (HuMAbs) were produced at Bristol-Myers Squibb, Seattle, WA by EB virus immortalization of 
peripheral blood lymphocytes from adult donors $(13,14)$. Cultures were screened for antibody against whole GBS and E. coli; positive cultures were cloned by limiting dilution. Nutrient-exhausted culture supernatant was concentrated by tangential flow ultracentrifugation. Antibodies were purified by affinity chromatography on a murine antihuman IgM MAb column. An antibody was produced from the peripheral B lymphocytes of an uninfected donor; it appears to react with all of the serotypes of GBS (13). A hybridomaproducing antibody that reacts with $\mathrm{K} 1 \mathrm{E}$. coli and group B meningococci was also detected (14).

The monoclonal and polyclonal antibody preparations were examined for opsonic activity using either standard phagocytosis and killing assays $(15,16)$ or a new flow-cytometric technique (16). Complement consumption was assessed by radial hemolytic assay in antibody containing agar plates (15).

The protective activity of the preparations against GBS and $E$. coli was examined in neonatal rats as previously described $(11,16)$. In a series of experiments, the effects of antibody administration on the inflammatory response in the peritoneal cavity was assessed (17) as well as the marrow neutrophil storage pool (metamyelocytes, bands, and segment neutrophils) by techniques previously described (18).

\section{Results}

The type III GBS MuMAb that we prepared was effective against both virulent and less virulent strains of type III GBS. The type-IIIspecific MuMAb protected $\geq 94 \%$ of the animals inoculated, regardless of whether the strains were of the more virulent variety, producing an excess of sialic acid containing type-specific capsular polysaccharide (7), or less virulent organisms (11). A MuMAb directed against the group $B$ carbohydrate also protected against two of the GBS strains $(23-36 \%)$, but the differences were not statistically significant. Doses as high as $750 \mathrm{mg} / \mathrm{kg}$ of IVIG had no protective effect against the more virulent strains, but did protect against the less virulent GBS strains (80-90\%). Thus, the MuMAbs were clearly more effective than the polyclonal human immunoglobulin preparations. 
Table 1

Protective Effect of Type III GBS MuMAb

\begin{tabular}{lcc}
\hline Time after inoculation & Number of animals & Survival rate, o \\
\hline No antibody & 17 & 0 \\
0 & 17 & 94 \\
$4 \mathrm{~h}$ & 15 & 87 \\
$8 \mathrm{~h}$ & 7 & 43 \\
$12 \mathrm{~h}$ & 20 & 25 \\
$24 \mathrm{~h}$ & 9 & 33 \\
\hline
\end{tabular}

${ }^{\mathrm{a}} \mathrm{Administered}$ at time intervals following bacterial inoculation of neonatal rats with $5 \times$ $10^{6}$ type 111 GBS.

Studies employing an IgG2a type-IIT-specific MuMab prepared by Egan and associates (12) showed that the MuMab IgM is approx 500 -fold more effective on a molar basis than the IgG MuMab in protecting against GBS infection in the neonatal rat. Protection was also observed when the antibody preparation was administered following bacterial inoculation (Table 1). Four hours after bacterial administration, the MuMAb continued to protect essentially as well as when it was administered at the same time as the bacteria. Protection was still significant when the MuMAb was administered 24 $h$ after inoculation.

Initial exposure of human neonates to GBS usually occurs through the respiratory tract. For this reason, we determined if the IgM could protect neonatal rats from respiratory infection. Following an intranasal inoculation of $1 \times 10^{7}-10^{8}$ type III GBS, only three of 64 untreated neonatal rats survived (11). In contrast, animals who received $1.8 \mathrm{mg} / \mathrm{kg}$ of the $\operatorname{IgM}$ MuMab by an intraperitoneal route had a survival rate of $94 \%$ with a $10^{7}$ and $77 \%$ with a $10^{8}$ intranasal bacterial inoculum (11). The IgM probably prevented invasion of the bacteria from the respiratory tract into the systemic circulation. We could not rule out the possibility that the IgM was actually getting onto the inflamed respiratory surfaces, however.

We next examined an IgA monoclonal antibody against the type III GBS antigen, which was prepared by colleagues at the University of Alabama (12). The IgA MuMAb also protected against systemic infection in the neonatal rat, but only when administered into the respiratory tract or systemically along with fibronectin (19). We have recently shown that this IgA monoclonal GBS antibody acts as an opsonin (20). This activity is significantly enhanced in the pres- 
ence of fibronectin (21). Moreover, the preparation is capable of triggering the complement system in rat but not in human serum (20).

The IgM GBS MuMAb, in a dose of $0.02 \mathrm{mg} / \mathrm{kg}$, markedly increased the influx of leukocytes into the peritoneal cavity of GBSinfected rats (17). The IgG2a MuMAb also enhanced leukocyte infiltration, but the dose required was much higher $(1.2 \mathrm{mg} / \mathrm{kg})$. The IgA GBS MuMAb did not enhance peritoneal leukocyte accumulation at any time and, in fact, suppressed the count four hours after bacterial inoculation. The IgM GBS MuMAb also prevented the severe neutropenia and depletion of marrow granulocyte stores commonly observed during GBS infection in the neonatal rat (18). The MuMAb of the IgM isotype has an absolute requirement for complement in providing functional opsonic and protective activity (22). We believe that the effect of the antibody on neutropenia and marrow neutrophil storage pool depletion is a result of complement activation and the release of leukocyte-mobilizing fragments of the third component of complement including $\mathrm{C} 3 \mathrm{~d}, \mathrm{~g}$ and $\mathrm{C} 3 \mathrm{e}$, as well as the chemoattractant C5a (23).

Administration of a murine antibody preparation to human neonates is unlikely to be approved, since reactions have been reported following the use of murine anti-CD3 antibody in adults. Raff and associates $(13,14)$ have recently developed at least two very unique human monoclonal antibodies. One of these appears (13) to react with all strains of group $B$ streptococci. These investigators (13) showed that this antibody is protective against type Ia and III GBS strains. We have studied the GBS HuMAb produced by Raff and colleagues in both protective and opsonic assays (16). As shown in Fig. 1, the HuMAb was effective in protecting neonatal rats from type III GBS infection in doses as low as $4 \mathrm{mg} / \mathrm{kg}$ (Fig. 1). The HuMab, at a dose of $20 \mathrm{mg} / \mathrm{kg}$, was significantly more effective in protecting neonatal rats from virulent type III GBS infection than was conventional IVIG (Sandoglobin ${ }^{\text {) }}$ in a dose of $500 \mathrm{mg} / \mathrm{kg}$ (Fig. 2). The GBS HuMab was also effective in similar doses against multiple strains of type Ia, II, and III GBS (Fig. 3). We have recently found that the IgM GBS HuMab is significantly more effective in both opsonizing GBS and activating the complement system than a genetically engineered IgG HuMab with identical Fab regions $(24,25)$.

Raff and associates (14) also produced a HuMAb reactive with the $E$. Coli $\mathrm{K} 1$ antigen and the group $\mathrm{B}$ meningococcal polysaccharide. This antibody is not reactive with non-K1 $E$. coli strains or 


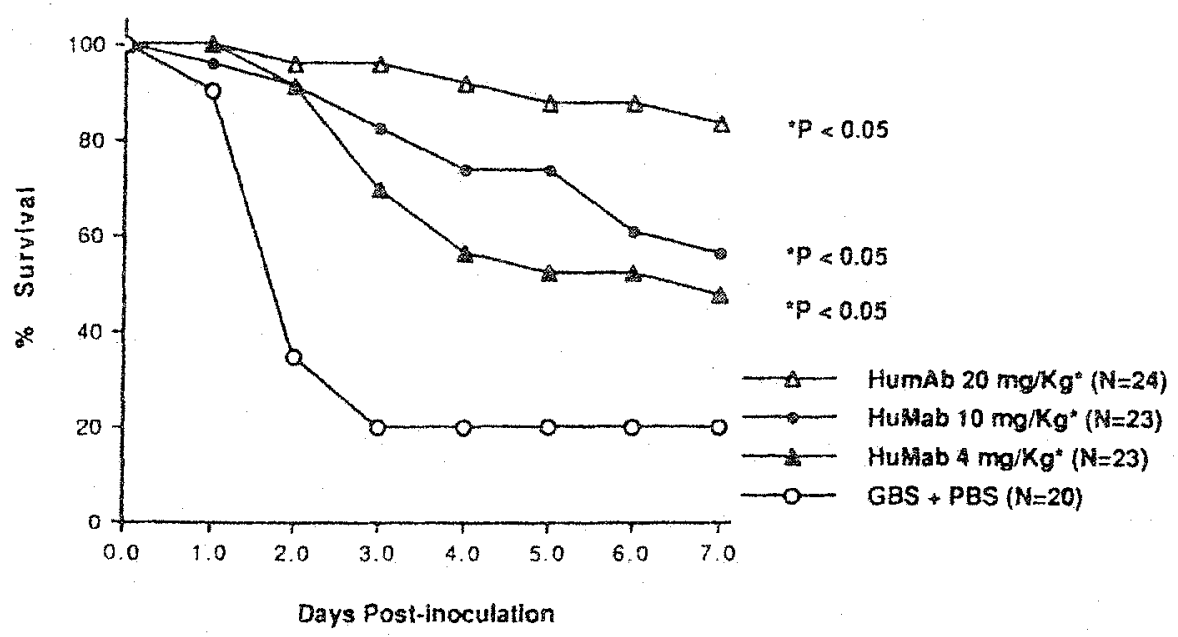

Fig. 1. Protective activity of varying doses of IgM human monoclonal GBS antibody against virulent type III GBS infection in neonatal rats. From Hill, $H$. R. et al. J. Infect. Dis. 163: 792-798, 1991, with permission from the University of Chicago Press, publisher.

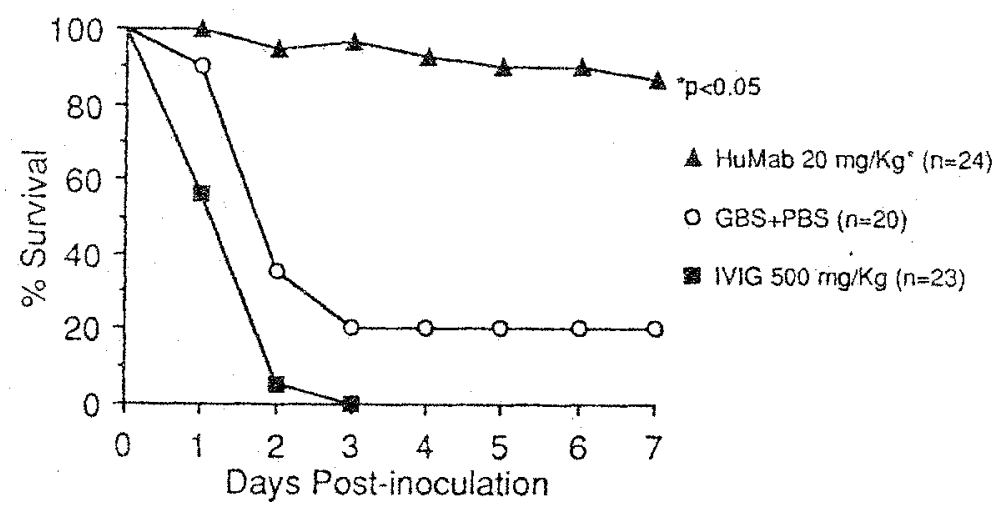

Fig. 2. Comparison of the protective effect of GBS HUMAb and conventional IVIG against type III GBS in neonatal rats. From Hill, H. R., et al., J. Infect. Dis. $163,292-298,1991$, with permission from the University of Chicago Press, Publisher.

other gram-negative organisms. The antibody possesses significant opsonic activity, but only in the presence of human complement. This HuMAb protected normal and neutropenic mice and neonatal rats from challenge with $K 1$ containing $E$. coli and Neisseria meningitides group $\mathrm{B}$ in doses ranging from 1 to $25 \mathrm{ug} /$ animal. 


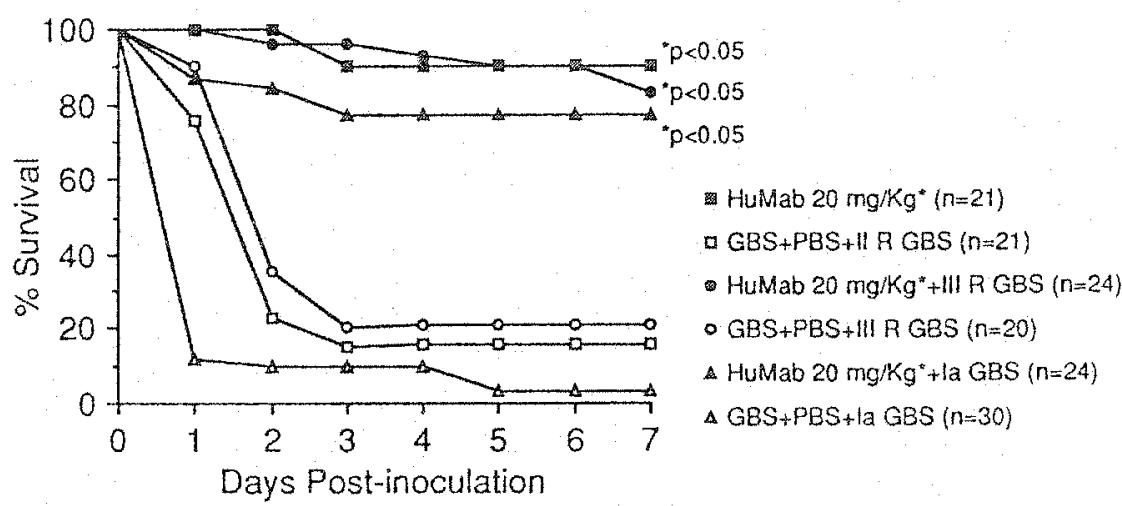

Fig. 3. Protective activity of IgM human monoclonal GBS antibody against virulent type la, II, and III GBS infection in neonatal rats.

\section{Discussion}

Monoclonal antibodies with striking opsonic and protective activity have been developed against GBS and K1 $E$. coli, two of the major bacterial pathogens affecting the human neonate. An IgM MuMAb that we developed was far more effective against all type III GBS strains than IVIG (11). This type-III-specific IgM monoclonal was approx 500-fold more effective on a molar basis than an IgG2a MuMAb directed against the identical epitope on the type III polysaccharide. The IgM antibody required complement for opsonic and protective activity (22) and affected the kinetics of leukocyte release from the marrow (18). We (23) reported that a fragment of $\mathrm{C} 3 \mathrm{~b}(\mathrm{C} 3 \mathrm{~d}, \mathrm{~g})$ promotes the release of neutrophils from the marrow, resulting in a peripheral neutrophilia in rats. Along with the local release of $\mathrm{C} 5 \mathrm{a}$, this likely results in a prompt accumulation of phagocytes at the site of bacterial invasion (17). We have shown that the ability of an antibody preparation to activate the complement system can be directly related to its protective and opsonic activity (15). IgM is much more active in triggering complement than IgG. This could aid in explaining the enhanced activity of our IgM MuMAb compared to the IgG2a MuMAb.

In contrast to the type specificity of the MuMAb, the HuMAb we studied was much more broadly reactive against all GBS organisms. Studies suggest that the HuMAb is directed toward the streptococcal group B polysaccharide, rather than type-specific po- 
lysaccharide antigens (13). Lancefield and coworkers (26) have suggested that antibodies to this cell-wall antigen, present on all group $B$ organisms, are not protective in a mouse model of infection. The protective activity of an antibody against GBS appears to be directly related to the avidity of the antibody for the antigen on the bacterial surface (27). Avidity is determined, in part, by the isotype of the antibody and the density of the epitope on the bacterial surface. Most of the data showing lack of protection with antibody directed against the group $B$ carbohydrate have been carried out with rabbit hyperimmune antiserum containing mostly IgG (26). Perhaps IgM, but not IgG, antibody directed against the group $B$ carbohydrate possesses sufficient avidity to result in opsonic and protective activity. The activity of an antibody preparation to combine with a bacteria and trigger the complement system is critical in its ability to afford protection (15). Such activation results in the generation of inflammatory mediators, such as $\mathrm{C} 3 e$ and $\mathrm{C} 3 \mathrm{~d}, \mathrm{~g}$, capable of releasing marrow granulocyte stores (23); C5a, which attracts leukocytes into the area of bacterial growth; and the deposition of $\mathrm{C} 3 \mathrm{~b}$ and iC3b on the bacterial surface. The IgM HuMAb was also approx 500 times more effective on a molar basis in opsonizing GBS and activating the complement system in the presence of these organisms. We suspect that this is one of the major reasons that both the IgM $M u M A b$ and the IgM HuMAb are so effective in protecting neonatal animals from lethal bacterial infection. Can IgM antibody be administered intravenously to humans without ill side effects? Haque and colleagues (28) have safely administered a preparation of IVIG to septic neonates enriched for IgM compared to standard IVIG. Moreover, human monoclonal anti-eJ5 IgM antibody in doses of 100 $\mathrm{mg}$ has been administered to adult humans without significant problems. Thus, it may be possible to safely administer GBS or E. coli K1 IgM HuMab to human neonates.

There is one major advantage of polyclonal preparations compared to monoclonal ones. Intravenous immunoglobulin is produced from plasma obtained from 2000 to as many as 10,000 donors. These preparations contain a broad antibody representation. Before one uses a very specific monoclonal, a firm diagnosis of the etiological agent based on antigen detection or culture would have to be made. A cocktail of HuMAb against GBS and E. coli K1 could be made for use in the human neonate. Such a preparation would not be effective against other gram-negative bacteria or against gram-positive 
organisms, such as Staphylococcus epidermidis, an increasingly common isolate from neonates. Most normal adult serum contains only low concentrations of antibody against GBS and $E$. coli $\mathrm{K} 1$, thus conventional IVIG preparations possess little activity against these pathogens. In contrast, standard IVIG contains excellent activity against Klebsiella pneumoniae, Serratia marcescens, Enterobacter, and staphylococci $(15,29,30)$. For this reason, a suitable strategy might be to add $\mathrm{HuMAb}$ against GBS and $E$. coli $\mathrm{KI}$ to conventional IVIG for use in septic neonates. The studies described here suggest the potential for the use of monoclonal antibodies, especially human monoclonal IgM antibodies, in the therapy of fulminant bacterial infections in the human neonate.

\section{ACKNOWLEDGMENTS}

We thank Nancy Augustine, Ann Shigeoka, Seth Pincus, John Bohnsack, Kuender Yang, and Robert Christensen, who contributed greatly to these studies. We also thank Jeannette Rejali for secretarial aid and Don Morse for illustrations.

Supported, in part, by US Public Health Service grant AI13150 and a grant from the Mead Johnson Research Center, Bristol-Myers Squibb Company.

\section{References}

1. Walsh, J. A. and Hutchins, S. (1989), Pediatr. Infect. Dis. J. 8, 271-276.

2. Hemming, V. G., Hall, R. T., Rhodes, P. G., Shigeoka, A. O., and Hill, H. R. (1976), J. Clin. Invest. 48, 1379-1387.

3. Baker, C. J. and Kasper, D. L. (1977), J. Infect. Dis. 136, S98-S104.

4. Shigeoka, A. O., Hall, R. T., and Hill, H. R. (1978), Lancet 1, 636-638.

5. Fischer, G. W., Hunter, K. W., Wilson, S. R., and Hemming, V. G. (1981), Lancet I, 271.

6. Santos, J. I., Shigeoka, A. O., and Hill, H. R. (1981), J. Pediatr. 99, 873879.

7. Shigeoka, A. O., Rote, N. S., Santos, J. I., and Hill, H. R. (1983), J. Infect. Dis. 147,856-863.

8. Yeung, M. K. and Mattingly, S. J. (1984), Infect. Immun. 44, 217-221.

9. Fischer, G. W. (1988), Pediatr. Infect. Dis. J. 7, S13-S16.

10. Galfre, G., Howe, S. C., Milstein, C., Butcher, G. W., and Howard, J. C. (1977), Nature 266, 550-552. 
11. Shigeoka, A. O., Pincus, S. H., Rote, N. S., and Hill, H. R. (1984), J. Infect. Dis. 149,363-372.

12. Egan, M. L., Pritchard, D. G., Dillon, H. C., Jr., and Gray, B. M. (1983), J. Exp. Med. 158, 1006-1011.

13. Raff, H. V., Sisscoe, P. J., Wolff, E. A., Maloney, G., and Shuford, W. (1988), J. Exp. Med. 168,905-917.

14. Raff, H. V., Devereux, D., Shuford, W., Abbott-Brown, D., and Maloney, G. (1988), J. Infect. Dis. 157, 118-126.

15. Yang, K. D., Bathras, J. M., Shigeoka, A. O., James, J., Pincus, S. H., and Hill, H. R. (1989), J. Infect. Dis. 159, 701-707.

16. Hill, H. R., Gonzales, L. A., Knape, W. A., Fischer, G. W., Kelsey, D. K., and Raff, H. V. (1991) J. Infect. Dis. 163,792-798.

17. Shigeoka, A. O., Weber, M. E., Pincus, S. H., Pritchard, D. G., Egan, M. L., and Hill, H. R. (1986), J. Infect. Dis. 153, 1170-1173.

18. Christensen, R. D., Rothstein, G., Hill, H. R., and Pincus, S. H. (1983), Pediatr. Res. 17, 795-799.

19. Hill, H. R., Shigeoka, A. O., Augustine, N. H., Pritchard, D., Egan, M. L., Lundblad, J. L., and Schwartz, R. S. (1984), J. Exp. Med. 159, 1618-1628.

20. Bohnsack, J. F., Hawley, M. M., Pritchard, D. C., Egan, M. L., Shigeoka, A. O., Yang, K. D., and Hill, H. R. (1989), J. Immunol. 143, 3338-3342.

21. Yang, K. D., Bohnsack, J. F., Hawley, M. M., Augustine, N. H., Knape, W. A., Egan, M. L., Pritchard, D. G., and Hill, H. R. (1990), J. Infect. Dis. 161, $236-241$.

22. Shigeoka, A. O., Jensen, C., Pincus, S. H., and Hill, H. R. (1984), J. Infect. Dis. 150,64-69.

23. Shigeoka, A. O., Gobel, R. J., Janatova, J., and Hill, H. R. (1988), Am. J. Pathol. 133, 623-629.

24. Raff, H. V., Bradley, C., and Brady, W. Comparison of functional activities between IgG1 and IgM class-switched hyman monoclonal antibodies reactive with group B streptococci or $E$ coli K1. (1991), J. Infect. Dis. 163, 346354.

25. Shyur, S. D., Raff, H. V., Bohnsack, J. F., Kelsey, D. K., and Hill, H. R. (1991), Comparison of the opsonic and complement activating activity of human monoclonal IgG and IgM antibody against group $B$ streptococci. Pediatr. Res. 29, 185A.

26. Lancefield, R. C., McCarty, M., and Everly, W. N. (1975), J. Exp. Med. 142, $165-179$.

27. Pincus, S. H., Shigeoka, A. O., Moe, A. A., Ewing, L. P., and Hill, H. R. (1988), J. Immunol. 140,2779-2785.

28. Haque, K. N., Zaidi, M. H., and Bahakim, H. (1988), Am. J. Dis. Child. 142, 1293-1296.

29. Hill, H. R. and Bathras, J. M. (1986), Rev. Infect. Dis. 8, S396-S400.

30. Yang, K D., Augustine, N. H., Gonzalez, L. A, Bohnsack, J. F., and Hill, H. R. (1988), J. Infect. Dis. 158,823-830. 